\title{
RHIC Prefire protection masks
}

\author{
A. Drees, C. Biscardi, T. Curcio, D. Gassner, \\ V. DeMonte, L. DeSanto, W. Fu, C.J. Liaw, \\ C. Montag, P. Thieberger, K. Yip
}

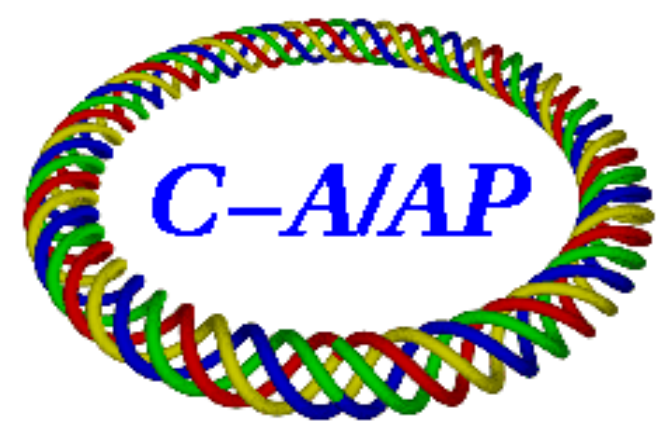

\section{Collider-Accelerator Department Brookhaven National Laboratory Upton, NY 11973}

\section{U.S. Department of Energy Office of Science, Office of Nuclear Physics}

Notice: This document has been authorized by employees of Brookhaven Science Associates, LLC under Contract No. DE-AC02-98CH10886 with the U.S. Department of Energy. The United States Government retains a nonexclusive, paid-up, irrevocable, world-wide license to publish or reproduce the published form of this document, or allow others to do so, for United States Government purposes. 


\title{
RHIC Prefire Protection Masks
}

\author{
A. Drees, C. Biscardi, T. Curcio, D. Gassner, V. DeMonte, \\ L. DeSanto, W. Fu, C.J. Liaw, C. Montag, P. Thieberger, K. Yip,
}

January 7, 2015

\section{Introduction}

The protection of the RHIC experimental detectors from damage due to beam hitting close upstream elements in cases of abort kicker prefires requires some dedicated precautionary measures with two general options: to bring the beam close to a limiting aperture (i.e. the beam pipe wall), as far upstream of the detector components as possible or, alternatively, to bring a limiting aperture close to the circulating beam. During the FY 2014 RHIC Heavy Ion run the first option was chosen because of the limited time available for preparation before the start of the run. For future runs the second option, in this case the installation of dual-sided movable masks, is preferred. The installation of the masks, one per ring, is planned before the start of the FY 2015 run.

\section{History}

Spontaneous and random prefires of abort kicker modules (Pulse Forming Network, PFN) have a history as long as RHIC is being operated. The abort system in RHIC consist of 5 kickers in per ring, each of them equipped with its own dedicated PFN. The kickers are typically operated with a voltage of about $27 \mathrm{KV}$ for $100 \mathrm{GeV}$ Au operation or $250 \mathrm{GeV}$ proton operation. With full voltage all five modules add a $1.6 \mathrm{mrad}$ angle to the beam trajectory, deflecting the beam safely into the dump.

\subsection{Prefires and Damage}

Since 2009 a total of 48 blue and 59 yellow prefires happened. Their distribution over the years and the PFNs is displayed in Tab. 1. The number per year varies greatly and so does the involvement of the PFNs. The perhaps only noteworthy characteristic appears to be the consistently small number of prefires caused by the middle PFN, number 3 . Out of the sum of 107 events, 44 occur during the ramp and a total of 9 without beam in the machine. Both the large number of events during ramps and the distribution over

\begin{tabular}{|c||c|c|c|c|c|c||c|c|c|c|c||c|}
\hline Ring & '09 & ' 10 & '11 & '12 & '13 & '14 & PFN1 & PFN2 & PFN3 & PFN4 & PFN5 & Sum \\
\hline \hline Blue & 11 & 12 & 9 & 2 & 8 & 6 & 13 & 9 & 2 & 13 & 9 & 48 \\
\hline Yellow & 2 & 8 & 15 & 8 & 14 & 12 & 3 & 16 & 5 & 14 & 20 & 59 \\
\hline
\end{tabular}

Table 1: Summary of blue and yellow prefires since 2009. 
the years is illustrated in Fig. 1. In Fig. 1 the time of a prefire is shown as a function of the fill number, thus spanning 6 years of operation as indicated and separated by the vertical dashed lines. The time is calculated with respect to the time of reaching flattop (ev-flattop) in a given ramp. Negative time values correspond to a prefire occurring before flattop is reached, i.e. during the ramp. $40 \%$ of prefires happen before flattop is reached. This is even more significant considering that, on average, much less time is spent ramping compared to the time spent at store and injection. Fig. 2 illustrates more details about

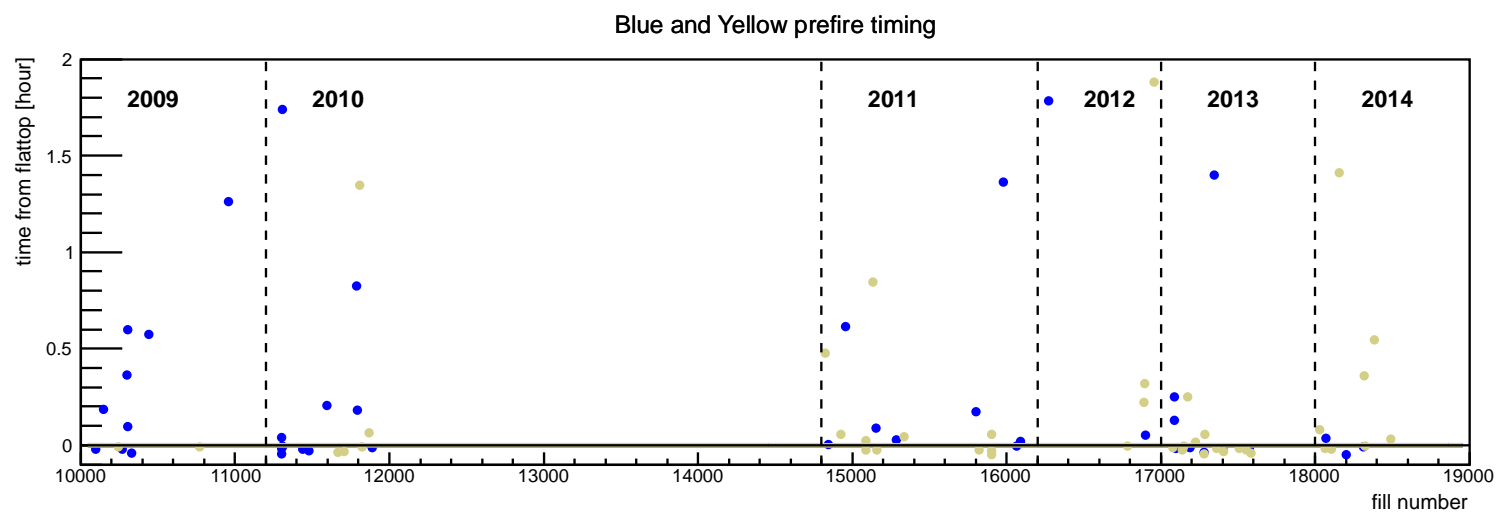

Figure 1: Prefire timing as a function of the fill number since 2009.

the timing distribution such as that $80 \%$ of all prefires happen within the first 2 hours of a fill with a mean value of 20 to 25 minutes (after ev-flattop) and an RMS of 40 to 50 minutes. The prefire with the lowest voltage occurred at $10.85 \mathrm{kV}$ in fill 10957 in 2009 ,
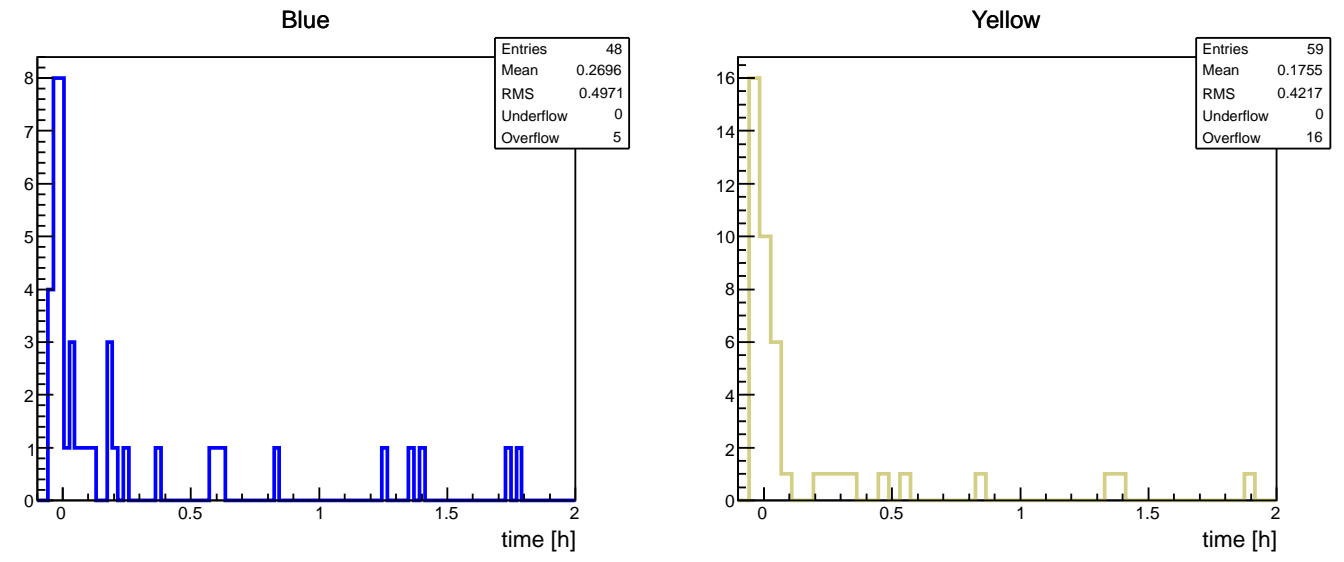

Figure 2: Number of prefire events as a function of time before or after flattop in the blue ring (left) and the yellow ring (right).

a run with $100 \mathrm{GeV}$ polarized protons. Coincidentally this is also the fill with the first reported damage to an experimental detector component.

A complete list of prefire incidents with damage to the STAR MTD electronics and the PHENIX MPC respectively is given in Tab. 2. The first MTD detector module was installed during the 2009 run on May 4th, the PHENIX MPC in 2006. No damage was reported from the PHENIX MPC prior to 2010, a year during which two incidents with 


\begin{tabular}{|c|c|c|c|c|}
\hline year & fill & species & intensity & comment \\
\hline 2009 & 10957 & pp $100 \mathrm{GeV}$ & $133 \cdot 10^{11}$ & $\begin{array}{l}\text { STAR MTD electronics is damaged } \\
\text { with the 1st blue prefire incident of the } \\
\text { run with the detector installed and on }\end{array}$ \\
\hline 2010 & 11593 & $\mathrm{AuAu} 100 \mathrm{GeV}$ & $113 \cdot 10^{9}$ & $\begin{array}{l}\text { STAR MTD electronics is damaged } \\
\text { with the 1st blue prefire incident with } \\
\text { the detector on }\end{array}$ \\
\hline 2010 & 11679 & $\mathrm{AuAu} 100 \mathrm{GeV}$ & $79 \cdot 10^{9}$ & $\begin{array}{lllll}\begin{array}{l}\text { PHENIX } \\
\text { channels }\end{array} & \text { MPC } & \text { detector } & \text { loses } & 5 \\
\end{array}$ \\
\hline 2010 & 11808 & $\mathrm{AuAu} 100 \mathrm{GeV}$ & $131 \cdot 10^{9}$ & $\begin{array}{l}\text { PHENIX MPC detector loses addi- } \\
\text { tional } 4 \text { channels }\end{array}$ \\
\hline 2012 & 16890 & $\mathrm{CuAu}$ & $90 \cdot 10^{9}$ & PHENIX MPC detector destroyed \\
\hline 2013 & 17347 & pp $255 \mathrm{GeV}$ & $102 \cdot 10^{11}$ & $\begin{array}{l}\text { STAR MTD electronics is damaged } \\
\text { with the 1st blue prefire incident of the } \\
\text { run (\# 17347) with the detector on }\end{array}$ \\
\hline
\end{tabular}

Table 2: Overview of blue and yellow prefires since 2009 leading to detector damage.

minor damage happened (losing 4 and 5 preamps respectively). In 2011 and 2012 none of the prefires happened while the MTD detector was powered preventing any damage. The quoted intensity in Tab. 2 is the total intensity of the offending beam at the time of the prefire. In the case of the MTD, detector damage always occured at the first "opportunity", i.e. the first prefire of a run with the detector powered. In the case of the PHENIX MPC the situation is not quite as simple. In 2010 there were 2 instances with rather minor damages and one instance in 2012 with major damage, losing 300 out of 416 crystal readout units, i.e. more than $70 \%$ of the MPC in this one event [4]. Coincidentially, it happened during the first heavy ion prefire incident in that year. It is not understood why other prefires before and/or after this event did not have the same destructive effect.

\section{$2.2 \quad$ Prefire Protection Bumps}

In order to prevent any further damage after the latest MTD incident in 2013 (see Tab. 2) and due to the lack of time for design, manufacturing and installation of dedicated prefire protection devices, the first of the two available options was chosen. The beam was brought close to the aperture by means of $20 \mathrm{~mm}$ horizontal orbit bumps in two arcs: sector 10 and 11 for the blue beam and sector 9 and 8 for the yellow beam. Fig. 3 shows the orbit bumps (design and measured orbit) for the blue ring.The second bump in sectors 11 (blue) and 8 (yellow) is for path length and dispersion compensation purposes. Equivalent bumps in Yellow sector 9 and 8 were installed in the yellow ring. Those bumps were present during the entire 2014 run and its 6 Blue and 12 Yellow prefire events. Comparison of loss patterns with and without prefire protection bumps should show the redistribution of the majority of losses away from experimental triplets towards the bump areas.

Fig. 4 indeed plainly demonstrates the positive effect of the protection bumps in case of a blue abort kicker prefire. While without the bumps the losses outside the dump area are concentrated in the STAR sector 5 triplet area, such losses are significantly reduced in the case with the bumps present. Losses are concentrated in the protection bump area with some others lighting up as well (such as collimators and compensation bump area) 


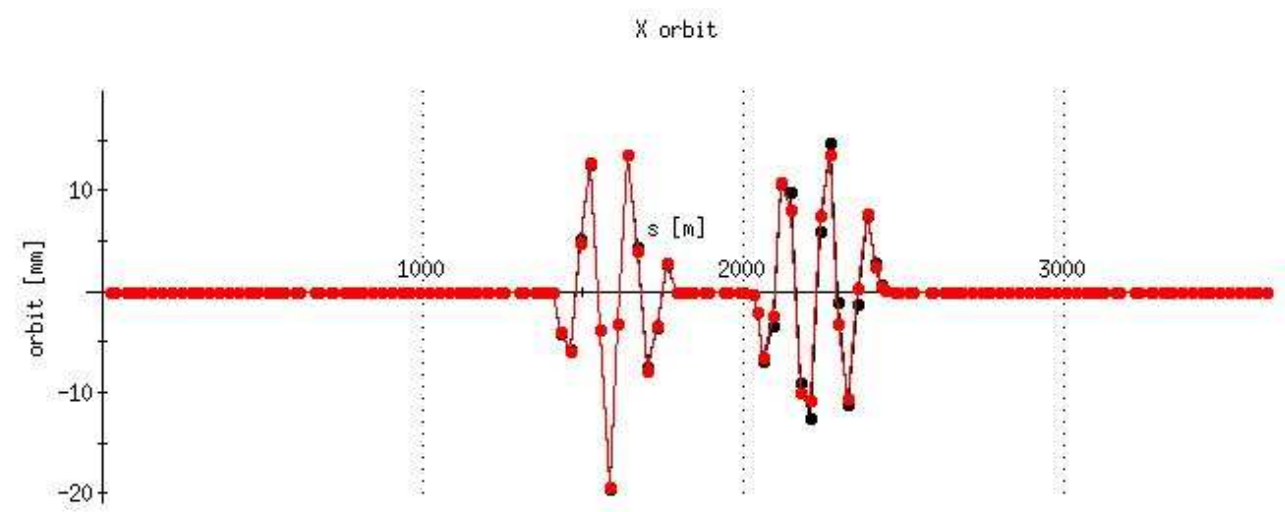

Figure 3: $20 \mathrm{~mm}$ orbit bumps installed in the blue ring for run 2014 prefire protection.
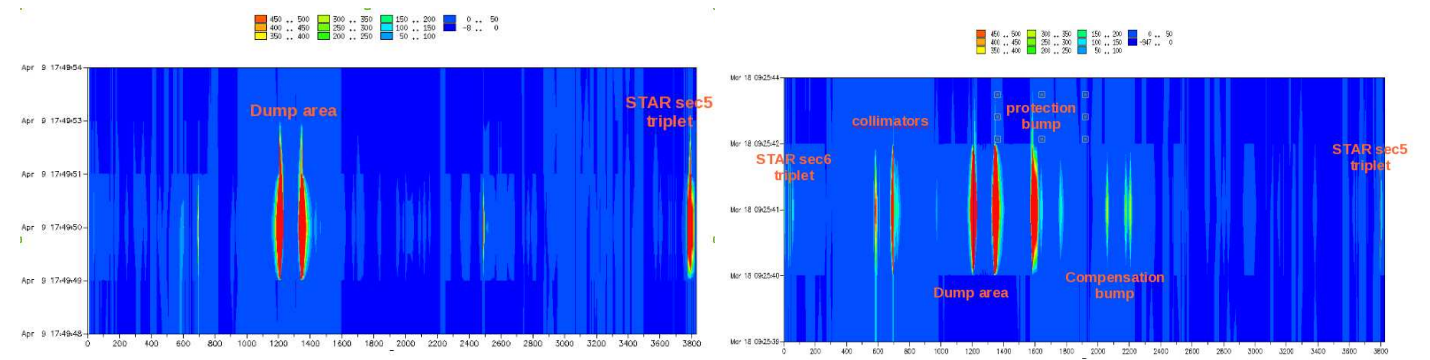

Figure 4: Left: Loss pattern after a blue abort kicker prefire in store 17347 (pp, run 13) without protection bumps. Right: after another prefire in store 18066 (AuAu, run 14) with protection bumps installed.

whereas STAR triplets are mostly spared. Given this success, one could argue to simply keep running with prefire protection bumps.

Unfortunately, besides absorbing beam during a prefire, the protection bumps had detrimental side effects. Increased continuous losses and subsequent increased radiation in the arcs caused FEC memory upsets and network switch resets in the alcoves [1]. Increased continuous losses, unusual for the center of an arc, are shown in Fig. 5. The figure shows loss patterns over 3 hours of a physics store in May 2014, during fill 18371. The red bands with losses of $50 \mathrm{rad} / \mathrm{hr}$ and above are created by the collimators placed around IP8. However, two other bands between $1500 \mathrm{~m}$ and $1600 \mathrm{~m}$ are also clearly visible. These two bands correspond to the Blue prefire protection bump area in sector 10. They clearly indicate continuous losses in that area of about 5-10 rads/hr. It is noticeable that no such losses are visible in sector 9 , the arc that hosts the yellow prefire protection bump.

Fig. 6 summarizes the number of network switch resets during the last 3 Heavy Ion runs. The immense increase in the number of network switch resets necessary in Run 14 compared to the earlier two runs is obvious. The increase is most significant for the 11B alcove which is the closest to the maximum of the blue protection bump. Sector 9 alcoves are located in the Yellow protection bump area.

In addition, the DX magnet shifts planned for 2015 cause significantly altered local orbits in the insertion areas. This in combination with a 90 degree F0D0 cell phase advance lattice needed for the ATS scheme (achromatic telescopic squeeze [2]) adds another layer of 


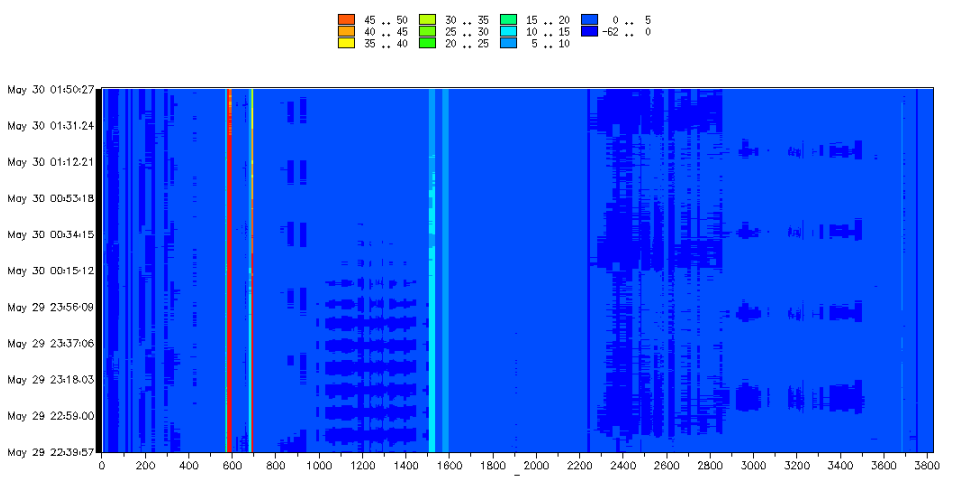

Figure 5: Loss pattern during a $100 \mathrm{GeV}$ AuAu store (18371) in run 14 with prefire protection bumps in place.

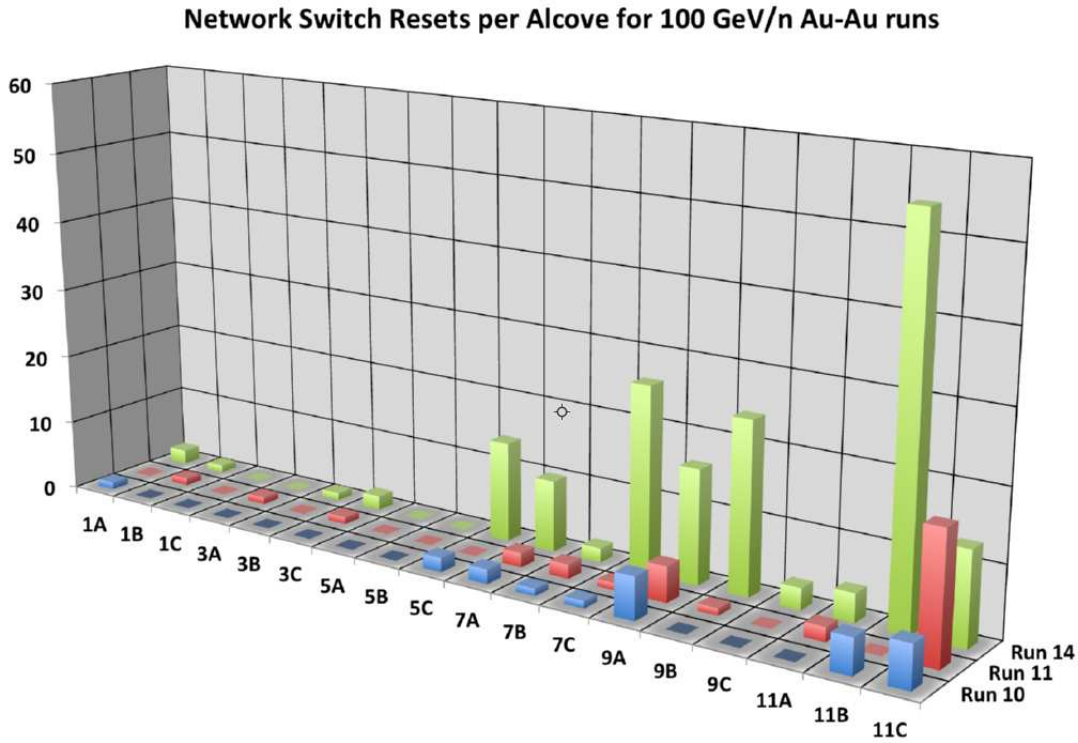

Figure 6: Network switch resets in RHIC alcoves during the last 3 heavy ion runs. Graph courtesy K. Brown [1]

complexity to the modeling effort that cannot be accomodated yet. The special lattice is required to provide a passive correction of non-linear chromaticities as well as to maintain a reasonable dynamic aperture. Both are essential in the quest to further improve RHIC's luminosity and are not compatible with $20 \mathrm{~mm}$ bumps in two arcs.

\section{Mask Design}

For a reasonable mask design aspects such as time schedule, availability, location, versatility and mask material had to be considered. 


\subsection{Location}

It would be most beneficial to install any prefire protection mask right in the beam abort area. Such a nearby installation would provide independence from lattice, phase advance and tune changes. At the same time, a warm space area with accessible beam pipe far enough away to allow for enough transverse deviation of the kicked beam from the closed orbit is needed. No such space is available downstream of the abort kickers in the same interaction region. The next available warm spaces are one arc away in both directions, i.e.

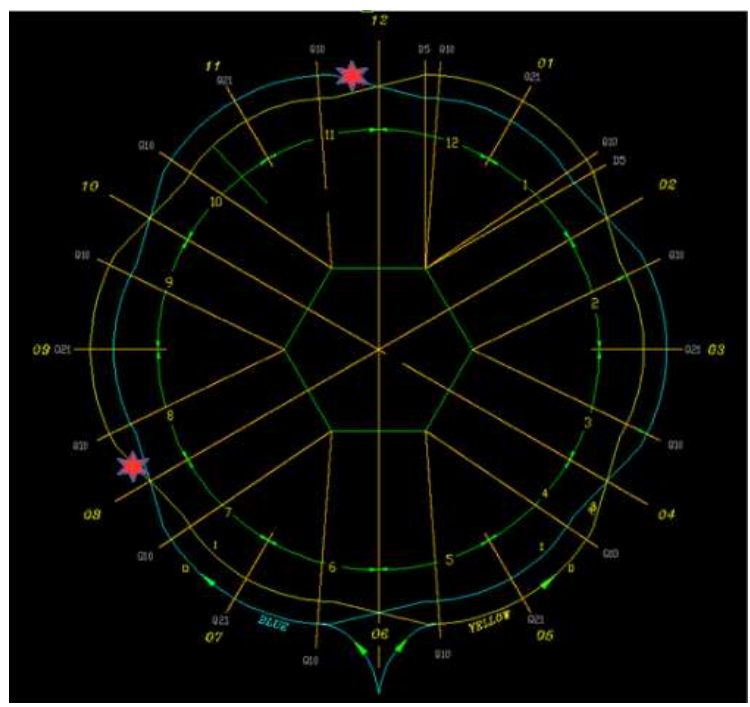

Figure 7: New mask locations in the RHIC rings (indicated by red stars).

between the Q4 and Q3 magnets on the sector 11 side of IP12 and sector 8 side of IP8. For the yellow ring no other location is possible since the PHENIX experiment is immediately down stream. Fig. 7 illustrates the chosen locations in RHIC. Due to already installed equipment in the opposite rings and the spin rotators in sector 8, the masks cannot be put right up to the Q4 magnets but need to be placed some distance away. This leads to a distance of $54.5 \mathrm{~m}$ in Yellow and of $65.6 \mathrm{~m}$ in Blue from the IP in question. Tunnel pictures and detailed drawings are provided in the Appendix (chapter A). No further equipment is installed between the masks and the Q3 magnets but there is further down stream, i.e. the PHENIX experiment in IP8 and the jet polarimeter in IP12. However, given the more than $50 \mathrm{~m}$ and $60 \mathrm{~m}$ distances, no additional shielding is required [5]. In fact, extra shielding close to the mask assemblies would provide material for particle showering and would not decrease radiation levels in the vicinity [5]. The configuration for the future mask is comparable to the radial material considered in [6]. Thus the maximum equivalent dose to an individual from an MCI (Maximum Credible Incident) is expected not to exceed $130 \mathrm{mrem}$ [5], [7]. An MCI is defined as one half of the beam, i.e. $2.5 \cdot 10^{13}$ protons, in a ring hitting a magnet at maximum considered beam energy $(300 \mathrm{GeV})$. Assuming the largest passable neutron flux in soil is $2 \cdot 10^{-6} \mathrm{~cm}^{-2}$ per proton, it takes $7.6 \cdot 10^{15}$ protons to reach the limit of $1000 \mathrm{pCi} / \mathrm{L}$ for tritium. This corresponds to more than 300 times the amount of beam considered in an MCI or 1267 times the amount considered for a prefire [5]. Even assuming an average of 10 prefires per ring and per year these numbers do not give cause for concern.

In order to be versatile and to be prepared for different possible lattices and phase advances (given that the chosen mask location is one arc away from the abort) the masks 
should be dual-sided. To allow for different beam sizes and a variety of RHIC operational modes such as low energy and injection they need to be movable and adjustable. This way continuous losses during stores (see Fig. 5) are also preventable. It turns out that

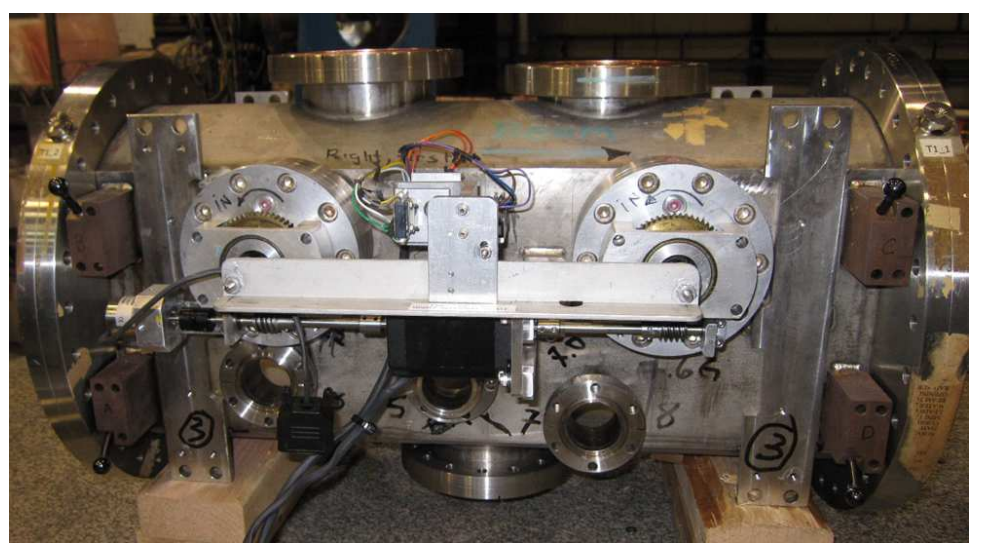

Figure 8: A FermiLab Style Cooling Tank on the bench before modification.

the retired FermiLab-style longitudinal stochastic cooling tanks provide all the necessary features and proportions to be turned into tanks for the prefire protection masks. Each tank is $77 \mathrm{~cm}$ long; 6 tanks are available, 2 of which will be used. Fig. 8 shows one of the existing tanks before modification. Obviously the inside of the tank needs to be modified, the stochastic cooling cavities need to be removed and mask jaws have to be inserted.

\subsection{Material}

One important input parameter for material heating and stress considerations is the maximum number of bunches affected by a prefire. Fig. 9 represents scope traces of the beam

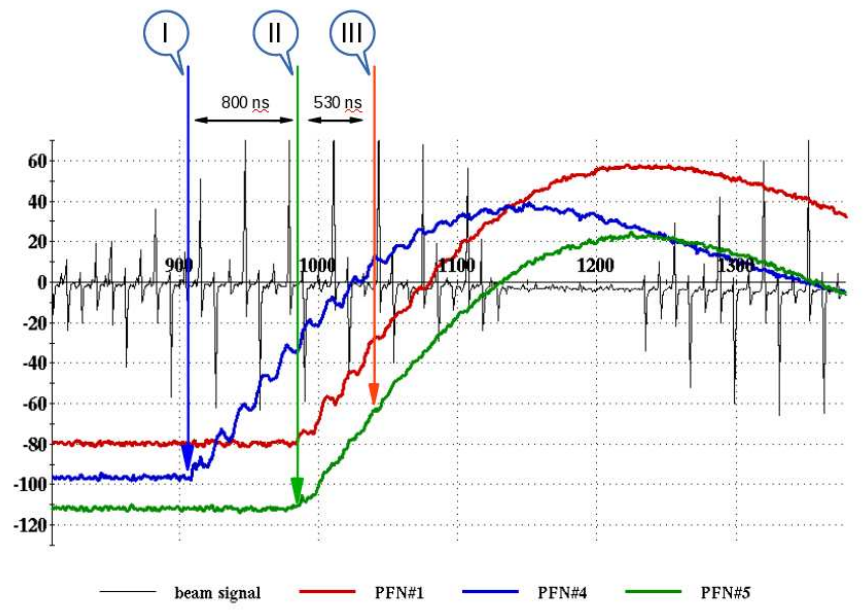

Figure 9: Blue abort kicker scope traces during a prefire ending store 18066 in run 14.The vertical scale is in arbitrary units, the horizontal scale corresponds to time in units of 10 ns.

signal and 3 PFN signals. The PFNs belong to the blue abort system. The data shows a 
snapshot from a prefire ending store 18066 in run 14. The three arrows, labeled I, II and III, indicate the following events:

(I) PFN\#4 prefires. The prefire can be identified by the early firing of one module in the middle of the bunch train signified by the presence of the beam signal (black trace).

(II) PFN\#1 and PFN\#5 follow approximately 800 ns later.

(III) Another 530 ns later, the sum of the three shown PFN signals reaches the nominal value encountered by the first bunch during an intended beam dump.

The total time lapsed between the start of the prefire and reaching the nominal value is approximately $1330 \mathrm{~ns}$, corresponding to about 13 bunches passing the abort kickers before a kick angle sufficiently large is reached to cleanly dump a bunch in the RHIC abort. This number is an already conservative estimate since the first few bunches will not receive enough of a kick angle to be intercepted by a mask or, likewise, a low beta triplet magnet. In addition, even a bunch with much less than the nominal deflection is supposed to hit the abort window and a deflection angle of as little as $0.82 \mathrm{mrad}$ is considered covered [8]. In this case, the minimum deflection is reached after about $1 \mu \mathrm{s}$ leading to only 10 affected bunches. However, in the following a conservative upper limit of 15 bunches to be intercepted by the mask material is assumed. The 15 affected bunches all receive different kick angles, starting at nearly zero and monotonically increasing to a nominal value of $1.6 \mathrm{mrad}$. Therefore they will spread horizontally over the mask surface as shown in Fig. 10. With 15 bunches superimposed as depicted here the maximum energy

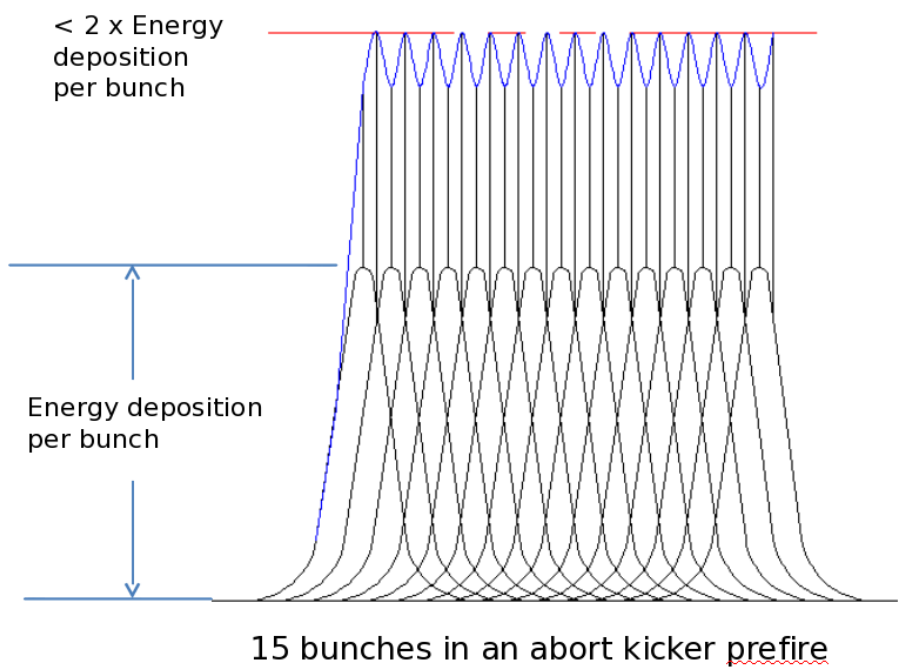

Figure 10: Sketch of energy deposition of 15 bunches distributed laterally on the mask material.

deposition on the surface corresponds to less than $2 \mathrm{x}$ the bunch intensity, as indicated by the horizontal red line in the sketch in Fig. 10. For our calculations we assumed an RMS beam size of $0.5 \mathrm{~mm}$, a per bunch proton intensity of $4 \cdot 10^{11}$ and a per bunch Au intensity of $2 \cdot 10^{9}$. Therefore heat and stress calculations were done for $8 \cdot 10^{11} \mathrm{p}$ and $4 \cdot 10^{9} \mathrm{Au}$. Fig. 11 shows one of the simulations done for $\mathrm{Au}$ beam with these parameters [9]. These 

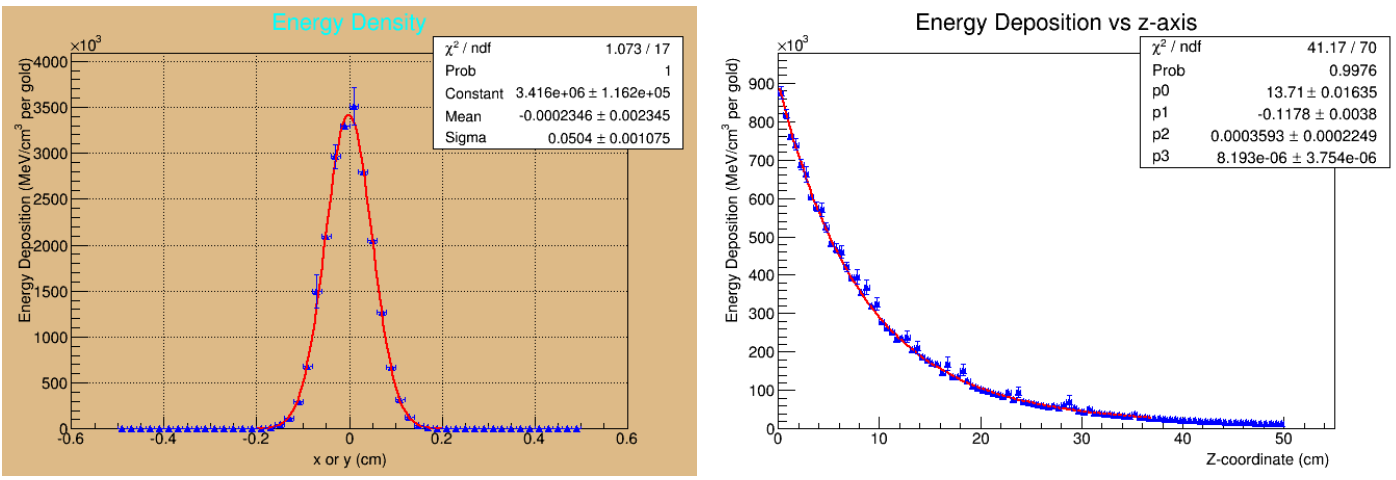

Figure 11: Energy deposition of Au beam on Ti (simulated).

energy density and deposition distributions are needed as input for the heat and stress simulations below.

We considered the following mask material candidates: stainless steel (SST 304), copper, Titanium (Ti-6Al-4V) and graphite. It turns out that proton beam impact would stay within limits even with 15 bunches for all materials while Au beams are more problematic causing over-heating (melting) or fracture of some materials (over-stressing). Tab. 3

\begin{tabular}{|c||c|c|c||c|c|c||c|c|}
\hline \multicolumn{1}{|c||}{} & \multicolumn{3}{c||}{ protons } & \multicolumn{3}{c||}{$\mathrm{Au}$} & \multicolumn{2}{c|}{ data sheets } \\
\hline Material & $\mathrm{N}_{\text {bunch }}$ & $\begin{array}{c}\mathrm{T}_{\max } \\
{\left[{ }^{\circ} \mathrm{C}\right]}\end{array}$ & $\begin{array}{c}\sigma_{\max } \\
{[\mathrm{MPa}]}\end{array}$ & $\mathrm{N}_{\text {bunch }}$ & $\begin{array}{c}\mathrm{T}_{\max } \\
{\left[{ }^{\circ} C\right]}\end{array}$ & $\begin{array}{c}\sigma_{\max } \\
{[\mathrm{MPa}]}\end{array}$ & $\begin{array}{c}\mathrm{T}_{\text {melt }} \\
{\left[{ }^{\circ} C\right]}\end{array}$ & $\begin{array}{c}\sigma_{\text {tensile }} \\
{[\mathrm{MPa}]}\end{array}$ \\
\hline \hline SST 304 & 15 & 542 & 379 & 1 & 511 & 370 & 1400 & 459 at $542^{\circ} \mathrm{C}$ \\
\hline & - & - & - & 2 & 962 & 399 & 1400 & 43 at $960^{\circ} \mathrm{C}$ \\
\hline & - & - & - & 15 & 6415 & - & 1400 & - \\
\hline Copper & 15 & 652 & - & 15 & 6967 & - & 1085 & 26 at $652^{\circ} \mathrm{C}$ \\
\hline Ti-6Al-4V & - & - & - & 1 & 394 & 243 & 1650 & 690 at $394^{\circ} \mathrm{C}$ \\
\hline & - & - & - & 2 & 709 & 464 & 1650 & 400 at $709^{\circ} \mathrm{C}$ \\
\hline Graphite & - & - & - & 2 & 580 & 16 & 3627 & 25.5 at $580^{\circ} C$ \\
\hline
\end{tabular}

Table 3: Heat and stress analysis (ANSYS) results for the considered materials.

summarizes the simulation results [10] for the considered materials. The stress limit of a material depends on the particular temperature reached in the impact resulting in decreasing stress limits with increasing temperatures. In cases of a temperature above the melting temperatures there is, obviously, no matching stress limit that could be quoted. Since protons are compatible with even the heaviest material (SST), no further simulations were done for the lighter ones. The decision to use the T-6Al-4V material rather than graphite is due to its higher material strength, higher melting temperature, easier machining, and higher density (which will therefore not be chipped off during the operation). Cutting graphite due to its dust production and dispersion is not handled at BNL. Similarly graphite causes concerns when installed inside the vacuum chamber. Beam power 
dissipation in two materials was modelled using Particle Studio [11]. 111 proton bunches of $3 \cdot 10^{11}$ p per bunch with an RMS bunch length of $20 \mathrm{~cm}$ were used in the model. The results are summarized in Tab. 4. Permeability is given normalized to empty space. None

\begin{tabular}{|l|c|c|c|}
\hline Material & $\begin{array}{c}\text { Resistivity } \\
{[\Omega \mathrm{cm}]}\end{array}$ & Permeability & $\begin{array}{c}\text { Dissipation } \\
{[\mathrm{W} / \mathrm{jaw}]}\end{array}$ \\
\hline SST 304 & $0.70 \cdot 10^{-4}$ & 1.008 & 0.20 \\
Ti-6Al-4V & $1.78 \cdot 10^{-4}$ & 1.00005 & 0.52 \\
Graphite & $8.50 \cdot 10^{-4}$ & 1.00 & 2.51 \\
\hline
\end{tabular}

Table 4: Electric properties of considered materials and resulting power depostion in the jaws.

of the studied materials would be cause for concern, i.e. there is no need for cooling of the jaws.

\subsection{Assembly}

Fig. 12 provides a drawing of the new assembly including the mask layout and dimensions. The angled mask surface along the beam direction allows for a grazing impact and helps to spread the bunches in the longitudinal direction. Each mask assembly holds two such jaws. The dimensions of the jaws were kept such as to not exceed the maximum weight limit on the weight bearing shafts. The weight of approximately $14 \mathrm{lbs}$ is significantly below that of the original stochastic cooling equipment (22 lbs).

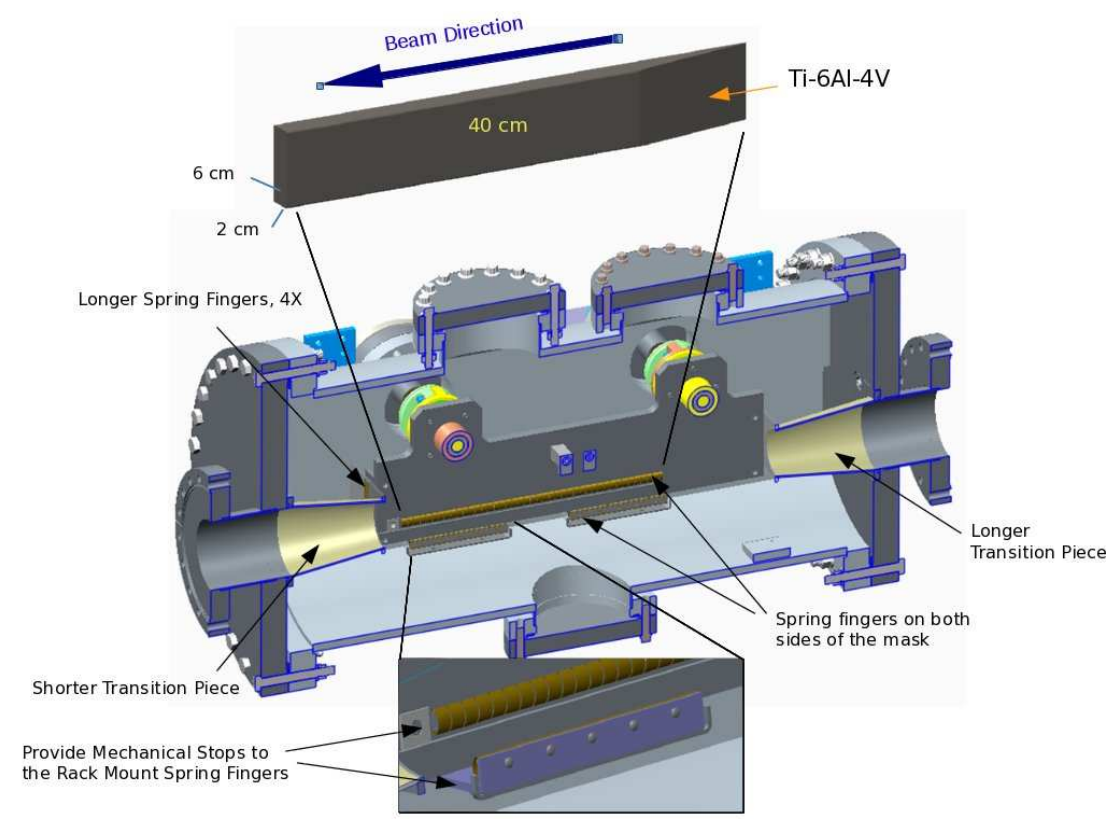

Figure 12: Y-Z cross section of the new mask assembly including jaw geometry and pointers to necessary modifications. 
After the modifications to the former stochastic cooling tanks the mask assembly's aperture will range from a minimum gap of $1 \mathrm{~cm}$ (store) to a maximum of $4.87 \mathrm{~cm}$ (injection). Each mask jaw is moved laterallyl and independently of the other by one stepping motor via two shafts. There will be no rotatry motion.

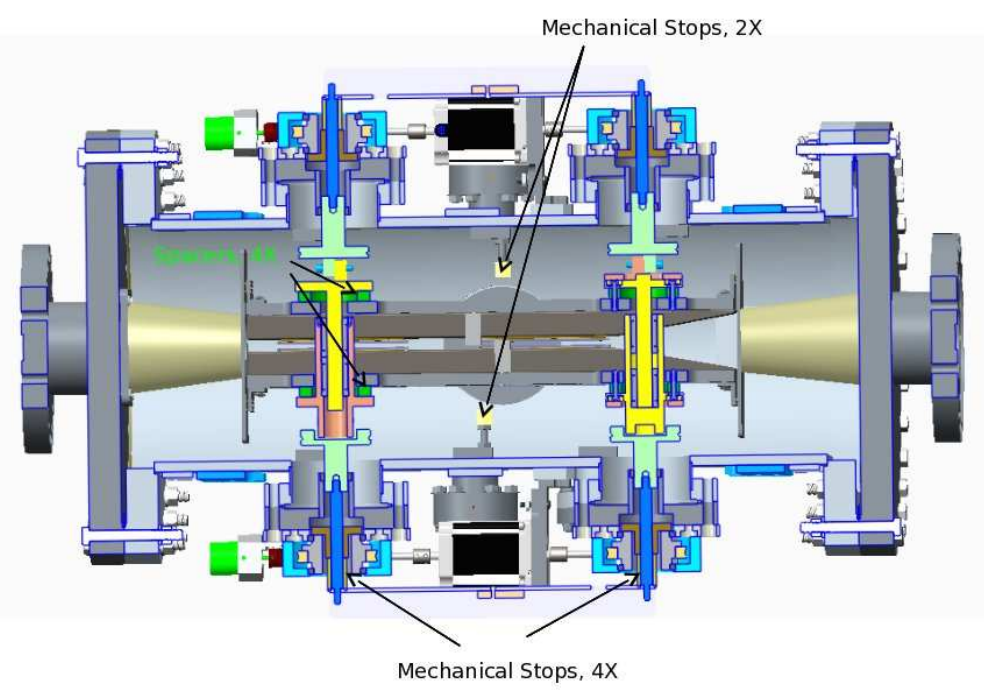

Figure 13: $\mathrm{X}-\mathrm{Z}$ cross section of the new mask assembly indicating the needed mechanical stops. View from the top.

Each mask assembly contains 2 stepping motors for independent motion control of the inside and the outside jaw. Pin diodes provide dedicated loss monitoring for setup and alarming purposes. Alarm levels have to be set by the system expert. Loss levels above alarm limit will create a software warning on the MCR alarm screen. There are two instances of identical software for mask operation, maskman.blue and maskman.yellow. A GUI similar to the collimator control application will be available for operation. The masks will be fully retracted each time beam is injected (4.87 cm aperture) and gradually moved in during the ramp once the abort kicker voltage exceeds $10.8 \mathrm{KV}$. This lower limit corresponds to the prefire incident with the lowest voltage to date (in 2009, see chapter 2.1). Minimal aperture is $\pm 0.5 \mathrm{~cm}$, centered around the center of the beam pipe by default. However, in order to allow a deviation of the closed orbit from the center of the beam pipe, the center position can be moved to the inside or outside within the allowable motion range. In this mode the controls software will move both jaws synchronously. For setup, however, each jaw can be moved independently. Each store the masks will move to a fixed and predetermined position. Positioning will be part of the overall accelerator ramping process ("tape sequences") once the masks and the managers are commissioned and the store positions are determined. Setup will be done with no more than 6 bunches in a particular ring. The desired positions contain 2 parameters (one position for each jaw) that have to be found during setup and stored. It is expected that the positions will have to be changed for each new lattice and ramp.

\section{References}

[1] Kevin Brown, private communication. 
[2] S. White, W. Fischer, Y. Luo, "Optics solutions for pp operation with electron lenses at $100 \mathrm{GeV} ", \mathrm{CAD}$ tech. note 2014.

[3] W. Zhang and J. L. Mi, private communication.

[4] X. He, RHIC Run 12 weekly meeting presentations, 05-29-2012, http://www.cad.bnl.gov/esfd/RMEM_12/rhic_planning.htm

[5] K. Yip, private communication.

[6] RSC Memo, 05-23-2012, "Equivalent Dose on the RHIC Berm from an MCI", http://www.c-ad.bnl.gov/esfd/RSC/Memos/rsc memos/main pages/2012_memos.htm

[7] D. Beavis, RSC chair, private communication.

[8] H. Hahn et al., "The RHIC Beam Abort Kicker System", PAC conference proceedings, NY 1999.

[9] MCNP6.1, T. Goorley et al., Initial MCNP 6 Release Overview, LA-UR-11-07082, Los Alamos National Laboratory, also Nuclear Technology, 180, pg 298-315 (Dec 2012).

[10] ANSYS simulation software, http://www.simutechgroup.com/Software/ANSYS/

[11] Particle Studio software, https://www.cst.com/products/cstps

\section{A Appendix}

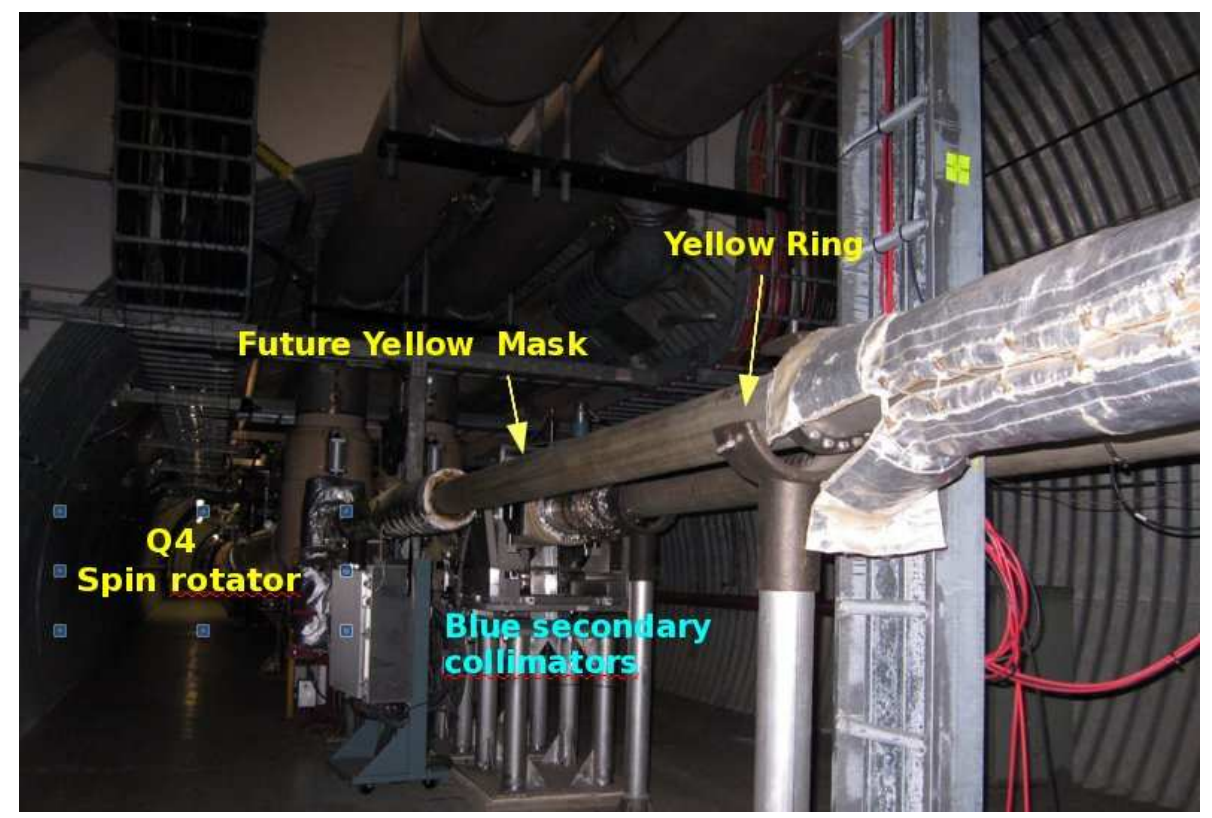

Figure 14: Picture of Yellow Installation Sector 8 


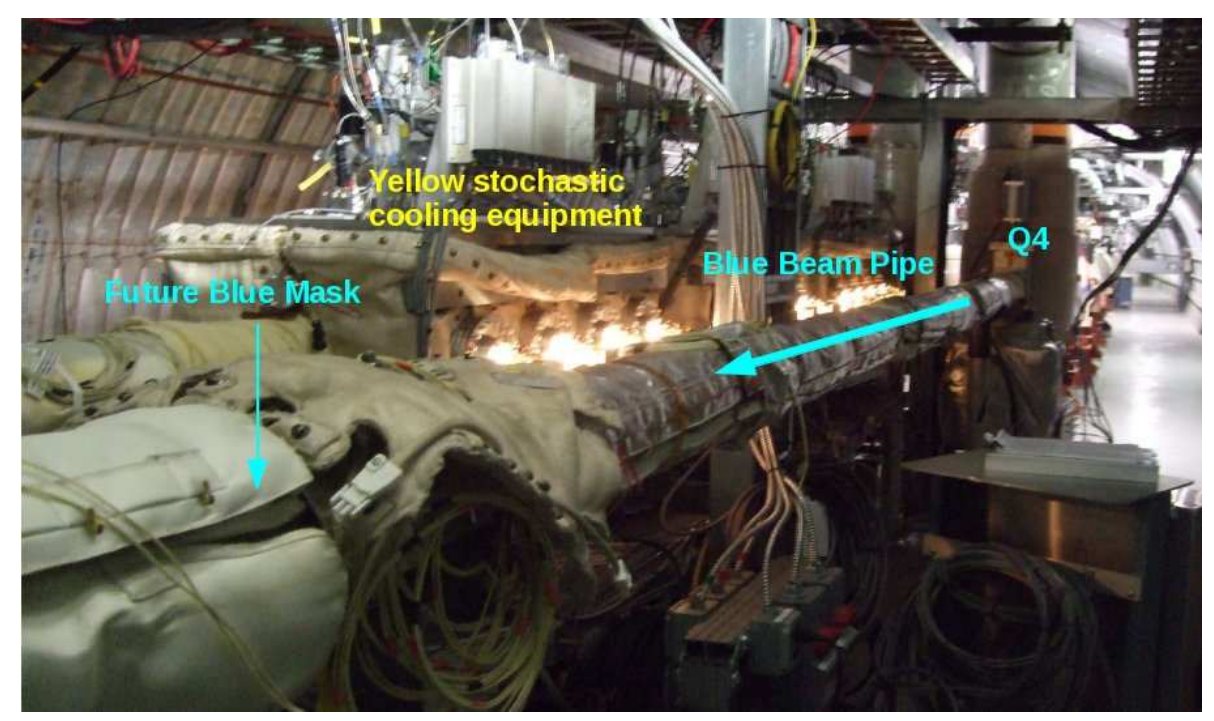

Figure 15: Picture of Blue Installation Sector 11 


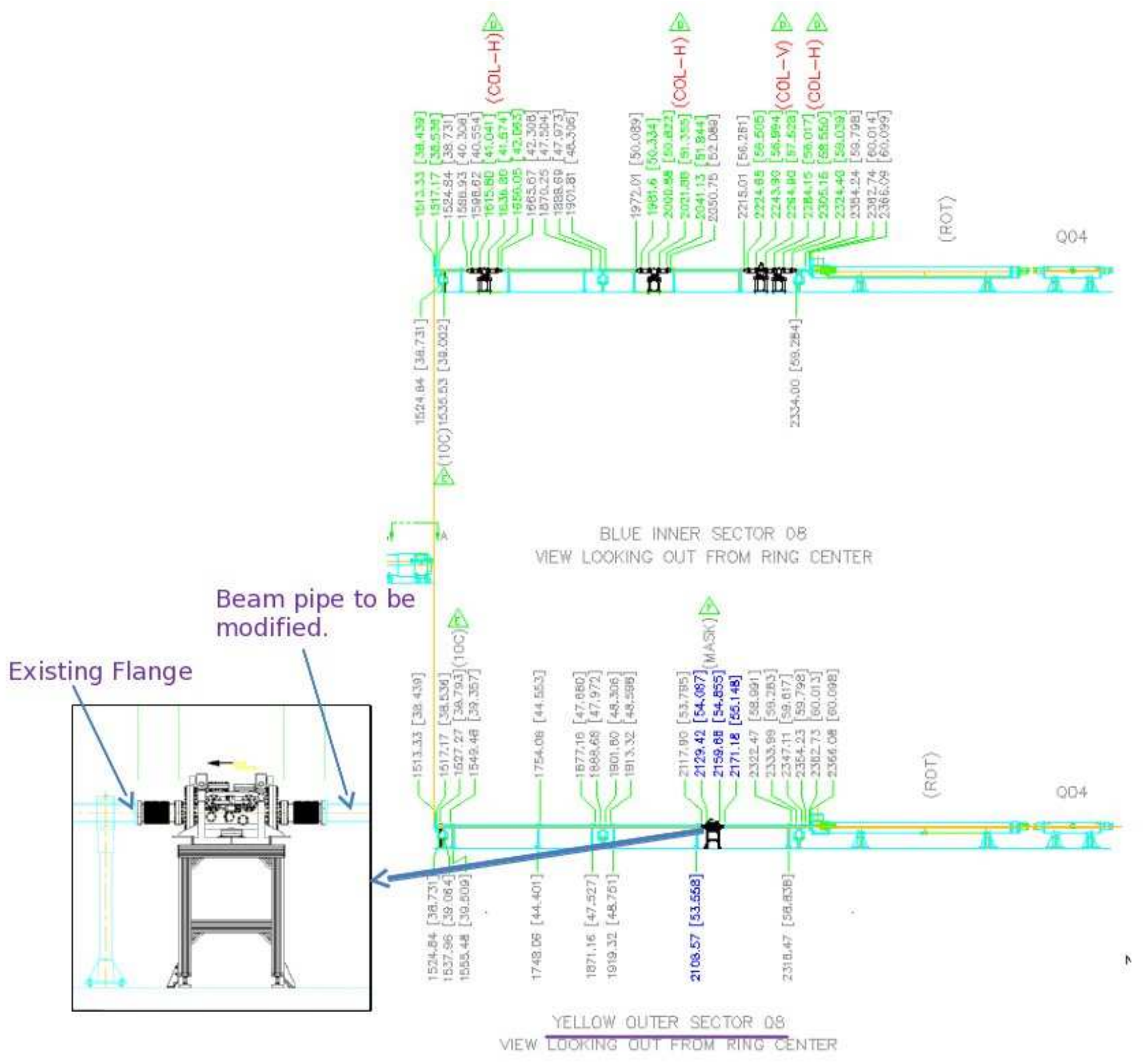

Figure 16: Drawing of Yellow Installation Sector 8 

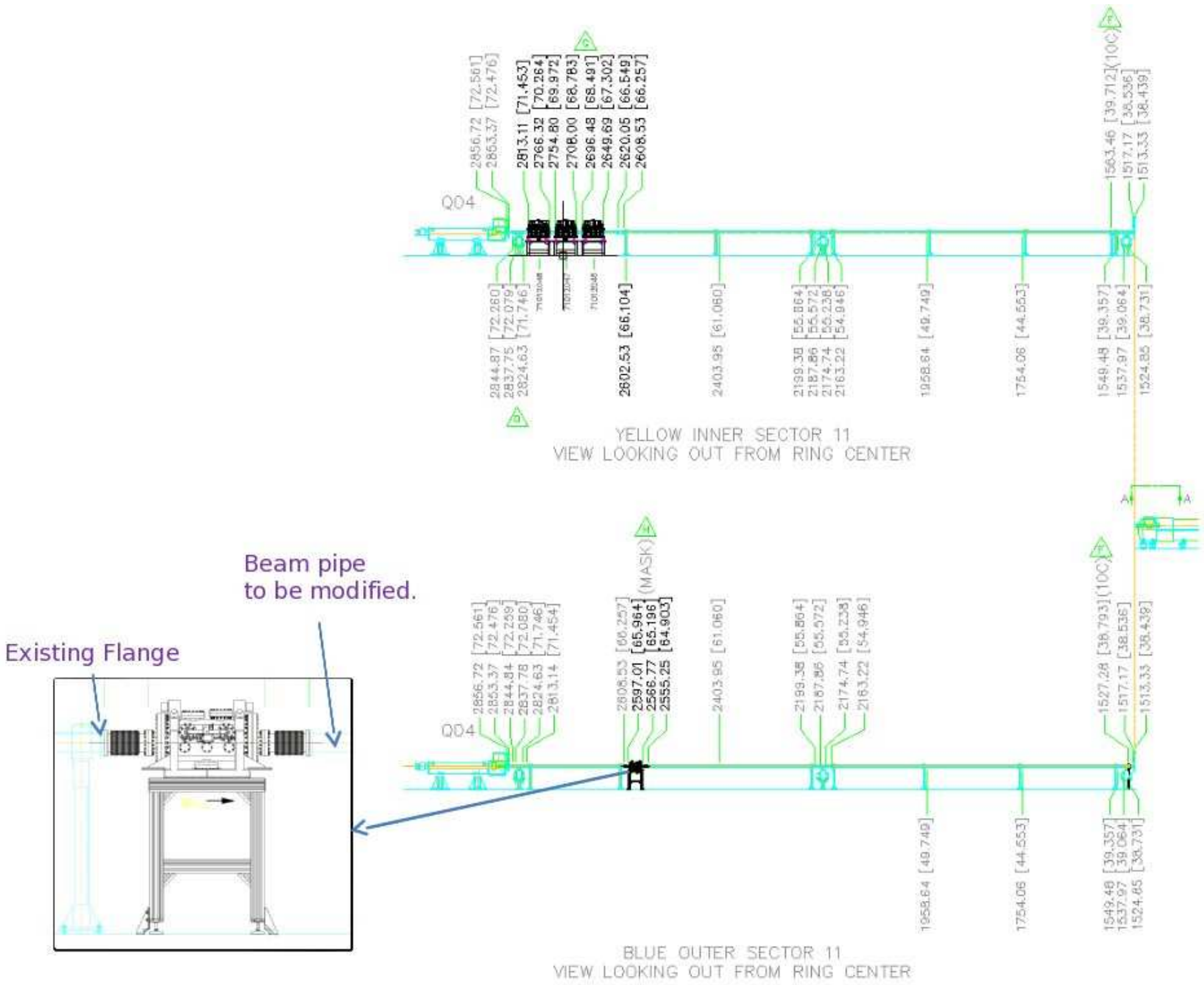

Figure 17: Drawing of Blue Installation Sector 11 